\title{
EDITORIAL
}

\section{GESTIÓN DE CUIDADOS}

Adela Zabalegui Yárnoz

Directora de Enfermería del Hospital Clinic de Barcelona.

Los retos en el cuidado de salud derivan del avance científico, los cambios demográficos, la crisis globalizada y el aumento de la demanda de servicios. El progreso en biología molecular y genética ha generado nuevos conocimientos y productos que aumentan las expectativas de mejora del cuidado generando mayor cronicidad. Los cambios demográficos, el envejecimiento e inmigración, en nuestro entorno redefinen este cuidado. Por otra parte, el aumento del coste del cuidado, en un contexto de crisis económica, incrementa la necesidad de un nuevo modelo asistencial basado en la eficiencia y en la competencia por los recursos asistenciales. A nivel global, existe una escasez de profesionales asistenciales, especialmente enfermeras/os, que hay que gestionar eficazmente. En nuestro país, el deseado cambio en la formación de Enfermería, mediante el paso de Diplomatura a Grado, desarrollará mayor competencia profesional y participación activa en el diseño de los nuevos modelos y sistemas de cuidados de la salud.

El aumento de la exigencia por parte de pacientes y familiares hacia el sistema sanitario queda reflejado en una mayor demanda de información y 
formación. Es necesario que incrementemos nuestra eficacia en el cuidado, centrado en el paciente y su familia, que se aplique con respecto, dignidad y empatía. Debemos informar, solucionar problemas de salud y ayudar a pacientes y profesionales en su toma de decisiones.

Nadie duda del compromiso de las enfermeras con el sistema de salud. La excelencia y prestigio de nuestros hospitales sólo se mantendrá si favorece el desarrollo profesional de sus enfermeras y otros profesionales. En el ejercicio profesional diario y en los procesos de cuidados, ofrecemos soluciones a los problemas de los pacientes y sus familias. Debemos, además, mostrar liderazgo facilitando la integración del cuidado en los diferentes niveles asistenciales y evaluando críticamente nuestra aportación. El diseño, difusión, aplicación y evaluación de nuevos modelos asistenciales requiere participación activa de todos los profesionales, pero en este contexto la enfermería debe ser "full partner" de los médicos en todo los procesos y tomas de decisiones.

Los gestores clínicos debemos implicar y transferir poder y responsabilidad a los profesionales asistenciales, enfermeras/os, médicos, ect. También, debemos reconocer, promover y visualizar la creatividad e innovación en la práctica del cuidado, a la vez que facilitar la aplicación de las competencias de enfermeras/os. La formación avanzada en enfermería conjuntamente con los nuevos roles de enfermería (por ejemplo las "Nurse practicioners" o las enfermeras de anestesia) han demostrado internacionalmente una gran eficacia y eficiencia. Deberíamos analizar los costes-eficacia de la posible aplicación de este tipo de enfermeras en nuestro sistema sanitario.

Nunca antes ha existido una oportunidad similar para potenciar la investigación en enfermería como elemento de desarrollo profesional y mejora del cuidado. Debemos generar conocimiento propio durante el ejercicio de nuestra actividad asistencial. Contamos ya, en nuestro país, con el Doctorado 
como nuevo marco de referencia para crear conocimiento en áreas concretas de interés. Ahora más que nunca, podemos mejorar el cuidado del paciente, aportando rigor científico a la solución de los problemas evidenciados en nuestro ejercicio profesional diario. Debemos afrontar este reto participando en equipos investigadores multidisciplinares, liderados por enfermeras o por otros profesionales, mostrando capacidad de colaboración dentro del equipo asistencial y realizando un trabajo individual meticuloso. El trabajo conjunto proporciona oportunidades de crecimiento personal y profesional a la par que facilita la consecución de los objetivos asistenciales.

Finalizo remarcando los elementos que fomentan el desarrollo profesional de la enfermería en nuestro entorno: primero el cuidado centrado en el paciente, familia y territorio; segundo el liderazgo basado en el desarrollo profesional dentro de un nuevo marco de competencias profesionales incluyendo los roles avanzados; tercero la solución de los problemas asistenciales mediante la evidencia científica y la investigación; y cuarto participación activa de la enfermería en todas las tomas de decisiones sobre el cuidado de la salud y el sistema sanitario.

Barcelona, 26 de enero del 2012 